\title{
Measuring verbal recall: A formula for chance estimation in serial position scoring'
}

JOSEPH F. FAGAN III AND LYNN M. LOWDEN

U.VIVERSITY OF CONNECTICUT

A formula for the expected value of chance scores in serial position scoring, $E_{M}\left(a_{i}\right)=n / N$, where $n=$ the number of correctly recalled items and $N=$ the number of serial positions to be filled, was presented and proven.

In the free recall situation, a S's response may be scored in a variety of ways. If, for example, the S's task were to recall the digits 5-8-4-3-1 and his response was 5-4-2-3-1, we may wish to measure simply the number of items recalled or we might be interested in the extent to which the order of the items has been reproduced. For theoretical reasons, investigators of memory processes (e.g., Conrad,1959; Bousfield \& Bousfield, 1966) find such a distinction between "item properties" and "order properties" of recall useful.

An integral part of scoring procedures for item and ordered recall is an estimate of what score would be expected were $S$ to respond randomly. Bousfield \& Bousfield have developed such formulas for item and ordered recall where ordered recall is defined as the number of correctly reproduced bigram sequences.

One other commonly used measure of ordered recall is a serial position score (SP), where one point is given for each item correctly recalled in the serial position it occupied in the order of presentation. $S$, in the example given above, has correctly recalled the digits 5,3 , and 1 in their correct serial positions and so would receive an uncorrected SP score of 3 points. The next step is to determine how many SP points $S$ would have received simply by guessing. Assuming that no correctly recalled items have been repeated and that all possible positions have been filled, the mean expected number of SP points by chance on a given item sequence, $E_{M}\left(a_{i}\right)$, is given by the following formula:

$$
E_{M}\left(a_{i}\right)=n / N
$$

where $\mathbf{n}$ is the number of correctly recalled items and $\mathrm{N}$ is the number of serial positions to be filled (or the number of items in the original message). For our hypothetical subject, $\mathrm{E}_{\mathrm{M}}\left(\mathrm{a}_{\mathrm{j}}\right)=4 / 5=.80$ and his corrected SP score $=3.00-.80$ or 2.20. It is also evident from Formula 1 that the value of $E_{M}\left(a_{i}\right)$ is always one or less.

The proof for Formula 1 is as follows:

Definitions:

$a_{1}=$ the occurrence of a particular correctly recalled item in the correct serial position. $a_{2}, a_{3} \ldots a_{n}=$ the occurrence of each of the remaining items in its correct serial position when there are n correctly recalled items.

$a_{i}=$ the occurrence of any one of the $n$ correctly recalled items in the correct serial position (each $a_{i}$ represents a point for position in our scoring system).

Proof:

Given that any single correctly recalled item may occur by chance with equal probability in any of $\mathrm{N}$ positions and that one and only one position is correct for any single correctly recalled item,

(1) The probability (p), then, that a particular correctly recalled item will be recalled by chance in the correct position is $1 / N$, or $p_{1}\left(a_{1}\right)=$ $p_{2}\left(a_{2}\right)=\ldots p_{n}\left(a_{n}\right)=1 / N$.

(2) Therefore, the expected number of chance occurrences of a particular correctly recalled item in the correct position for $\mathrm{k}$ randomly drawn (chance) recall sequences, $E_{k}$, is $1 / \mathrm{N}$. $\mathrm{k}$ or $\mathrm{k} / \mathrm{N}$.

(3) With $n$ such items, the expected number of chance occurrences of any correctly recalled item in the correct position for $\mathrm{k}$ chance recall sequences is the sum of the $E_{k^{s}}$ for each of $n$ correctly recalled items, or

$$
E_{k}\left(a_{1}\right)+E_{k}\left(a_{2}\right) \ldots+E_{k}\left(a_{n}\right)=E_{k}\left(a_{i}\right)
$$

(4) Since $E_{k}$ for a particular correctly recalled item $=\mathrm{k} / \mathrm{N}$ (from Statement 2), by substitution in the equation in Statement 3 we see that $E_{k}\left(a_{i}\right)=k n / N$.

(5) The mean expected number of chance occurrences of any of $\mathbf{n}$ correctly recalled items in the correct position, $\mathrm{E}_{\mathrm{M}}\left(\mathrm{a}_{\mathrm{i}}\right)$, for a single randomly drawn sequence, then, is $[\mathrm{kn} / \mathrm{N}] / \mathrm{k}$, or $E_{M}\left(a_{i}\right)=k n / N \cdot 1 / k=n / N$.

\section{References}

Bousfield, A. K., \& Bousfield, W. A. Measurement of clustering and of sequential constancies in repeated free recall. Psychol. Rep., 1966, 19, 935-942.

Conrad, R. Errors of immediate memory. Brif. J. Psychol., 1959, 50, 349-359.

\section{Notes}

1. This investigation was supported (in part) by a Public Health Service Training Grant (5T-01-HD0084-03) from (National Institute of Child Health and Human Development) Public Health Service to the senior author. Formula 1 was developed by the senior author and the prosf was derived by the junior author. 